\section{Resultados de pacientes con linfoma del manto: impacto de terapias basadas en citarabina y trasplante hematopoyético}

\author{
JAMES CAMPBELL ${ }^{1,2}$, SEBASTIÁN HURTADO ${ }^{1, a}$, CONSUELO KUTZ ${ }^{1, a}$, \\ KATHERINE SOTO ${ }^{1,2}$, DANIEL ERNST ${ }^{1,2,3}$
}

\section{Cytarabine and hematopoietic cell transplantation for mantle cell lymphoma. Analysis of 20 patients}

Background: Mantle cell lymphoma (MCL) has high relapse and mortality rates. There is a survival benefit when treatment is intensified with cytarabine (AraC), hematopoietic cell transplantation (HCT) and maintenance with rituximab. Aim: To assess the outcomes of patients with MCL treated in a university hospital. Material and Methods: Review of an oncology center database and medical records identifying patients with MCL treated between 2006 and 2017. Death dates were obtained from the death certificate database of the National Identification Service. We analyzed the response rate, overall survival (OS) and progression-free survival (PFS). As a secondary objective, the survival impact of AraC, HCT and maintenance with rituximab, was also analyzed. Results: Information on 20 patients aged $62 \pm 11$ years, followed for a median of 45 months was retrieved. Eighty-five percent were diagnosed at an advanced stage. The most used first-line regime was $R$-CHOP in 11 patients, followed by $R$-HyperCVAD in five. Only 47\% achieved complete response. 4-year PFS and OS were of 30 and $77 \%$ respectively. Mantle Cell Lymphoma International Prognostic Index (MIPI) significantly predicted PFS and OS. Maintenance with rituximab or HCT was associated with better PFS (48 vs 21 months, $p<0.01$ ). The exposure to AraC or HCT, in refractory or relapsed disease, was associated with an increase in PFS from 9 to 28 months $(p=0,02)$ and 4-year OS from 40 to $100 \%$ ( $p=0.05)$. OS increased even more, from 25 to $100 \%$ in those with high-risk MIPI ( $p=0.04)$. Conclusions: The incorporation of AraC, HCT and maintenance with rituximab in the therapeutic backbone of $M C L$, especially for high-risk cases, was associated with improved survival.

(Rev Med Chile 2019; 147: 9-17)

Key words: Lymphoma, Mantle-Cell; Cytarabine; Hematopoietic Stem Cell Transplantation; Rituximab; Chile.
${ }^{1}$ Escuela de Medicina, Facultad de Medicina, Pontificia Universidad Católica de Chile. Santiago, Chile. ${ }^{2}$ Departamento de Hematología y Oncología. Hospital Clínico, Red de Salud UC-Christus. Santiago, Chile.

${ }^{3}$ Instituto de Ingeniería Biológica y Médica. Pontificia Universidad Católica de Chile. Santiago, Chile. ${ }^{\text {a} A l u m n o ~ d e ~ l a ~ C a r r e r a ~ d e ~}$ Medicina.

Trabajo no recibió

financiamiento.

Los autores declaran no tener conflictos de interés.

Recibido el 13 de septiembre de 2018, aceptado el 27 de diciembre de 2018.

Correspondencia a: Daniel Ernst Diagonal Paraguay $362,6^{\circ}$ piso Departamento de Hematología y Oncología. Escuela de Medicina, Facultad de Medicina. Pontificia Universidad Católica de Chile. Santiago, Chile. dmernst@uc.cl e los linfomas no Hodgkin (LNH) de células B, el linfoma del manto (LM) representa 5\%-8\% y normalmente se asocia a mal pronóstico ${ }^{1-3}$. Los pacientes con LM siguen considerándose incurables y la mediana de sobrevida no suele superar los 5 años $^{4-6}$.

El LM se caracteriza por presentarse usualmente en etapa IV (80-90\%), comprometer tracto 
digestivo, bazo y médula ósea y tener una baja respuesta a tratamiento ${ }^{7-9}$. En los primeros estudios clínicos se incluyeron a los pacientes con LM junto a aquellos con linfoma difuso de células grandes $\mathrm{B}$ (LDCGB), derivando en que CHOP (ciclofosfamida, doxorrubicina, vincristina y prednisona) y luego R-CHOP (CHOP más rituximab) se estableciese como la terapia estándar ${ }^{10,11}$. Con este esquema de quimioterapia, $94 \%$ de los pacientes menores de 65 años presenta respuesta completa (RC) o parcial (RP) al tratamiento y $34 \%$ de estos logra RC. Sin embargo, a 2 años, más de la mitad de los pacientes recae y $24 \%$ fallece.

En la última década, numerosos estudios han demostrado que los pacientes con LM se benefician de una terapia intensificada ${ }^{12-17}$. Particularmente, los esquemas que han demostrado beneficio en sobrevida son el trasplante de precursores hematopoyéticos $(\mathrm{TPH})$ y protocolos de alta intensidad, que incluyen al análogo de nucleósido citarabina (AraC). Más recientemente, se demostró el beneficio de la terapia de mantención con rituximab por 2 años luego de la quimioterapia o $\mathrm{TPH}^{18}$. Por otro lado, numerosas nuevas drogas (bortezomib, lenalidomida, ibrutinib) han probado ser activas y están aprobadas para enfermedad recaída o refractaria ${ }^{19-21}$, pero en ningún caso su beneficio logra el impacto de los esquemas anteriormente mencionados en primera línea. Por este motivo, las guías internacionales actuales recomiendan incluir los tratamientos con AraC, TPH y mantención con rituximab como pilares del tratamiento, siempre que las condiciones del paciente lo permitan, e idealmente en primera línea ${ }^{22}$.

En Chile no hay datos publicados respecto a los resultados de los pacientes con LM tratados con quimioterapia ni TPH. Por lo anterior, revisamos retrospectivamente los resultados de los pacientes con LM tratados en el Centro de Cáncer y Hospital Clínico UC-Christus, y evaluamos el impacto en respuesta a tratamiento y sobrevida que el uso de TPH, AraC y mantención con rituximab aportan.

\section{Pacientes y Métodos}

\section{Diseño del estudio y selección de pacientes}

Estudio observacional, analítico y retrospectivo. Se estudiaron pacientes con diagnóstico de LM atendidos en el Centro de Cáncer y Hospital
Clínico de la Red de Salud UC-Christus entre enero de 2006 y julio de 2017. Se incluyeron en el análisis a pacientes con diagnóstico confirmado mediante biopsia de tejido, de acuerdo a la clasificación de la Organización Mundial de la Salud de $2008^{23}$, revisado en caso de diagnóstico previo a dicho año; o por citometría de flujo en casos con biopsia no concluyente o compromiso aislado de sangre periférica. Además, debieron haber recibido al menos una línea de tratamiento completa (u observación activa) y contar con al menos tres meses de seguimiento a contar del diagnóstico. La información fue obtenida de las bases de datos del Centro de Cáncer de la Red de Salud UC-Christus, del Departamento de Hematología y Oncología UC y mediante revisión de la ficha clínica electrónica institucional. Las fechas de defunción fueron obtenidas de la página web del Registro Civil (www.srcei.cl). Previo al análisis de los datos, la identidad de los pacientes fue omitida, según recomendación del Comité Ético Científico UC.

\section{Objetivos y variables estudiadas}

El objetivo principal fue analizar los resultados de los pacientes con LM tratados en nuestra institución, incluyendo tasa de respuesta, sobrevida global (SG) y sobrevida libre de progresión (SLP). Objetivos secundarios fueron analizar el impacto pronóstico de la estratificación de riesgo según índice MIPI y diferencias en sobrevida entre pacientes tratados con o sin AraC, TPH y mantención con rituximab. La respuesta a tratamiento fue determinada según la clasificación de Lugano en $2014^{24}$. Se consideraron las variables demográficas de los pacientes, etapa de presentación según la clasificación modificada de Ann-Arbor ${ }^{25}$, índice de riesgo MIPI, los diferentes protocolos de quimioterapia, líneas de tratamiento, indicación de TPH y/o mantención con rituximab $\left(375 \mathrm{mg} / \mathrm{m}^{2}\right.$ intravenoso cada 3 meses por 2 años). Se analizó sobrevida mediante el método de Kaplan-Meier y las diferencias se estimaron según método de log-rank o Gehan-Breslow-Wilcoxon, según correspondiera; las diferencias en variables no paramétricas se analizaron con test $\chi^{2}$ y prueba $U$ de Mann-Whitney, según correspondiera; y las diferencias en variables paramétricas mediante prueba t de Student. El análisis fue realizado con el software GraphPad Prism 7. Se consideró significativo un valor $\mathrm{p}<0,05$. 


\section{Resultados}

\section{Pacientes y esquemas de tratamiento}

En los períodos descritos, 23 pacientes con LM fueron tratados en nuestra institución. De estos, 2 fueron excluidos por seguimiento menor a 3 meses y 1 por continuar tratamiento en otro centro. Por lo tanto, se analizaron un total de 20 pacientes. La mediana de seguimiento fue de 44,7 meses. La edad al momento del diagnóstico fue de $62 \pm 11$ años y en su mayoría se presentaron con enfermedad en etapa IV (80\%). De los pacientes en etapa IV, todos tuvieron compromiso de médula ósea (Tabla 1). En 19 casos se contó con biopsia que confirmó el diagnóstico de LM, mientras que en el paciente restante el diagnóstico se realizó por citometría de flujo en sangre periférica.

Tabla 1. Aspectos demográficos, presentación clínica al momento del diagnóstico y tratamiento en primera línea

\begin{tabular}{|ll|}
\hline Linfoma del manto & $\mathrm{n}=20$ \\
\hline Edad (años, media \pm DS) & $62 \pm 11$ \\
\hline Sexo masculino (\%) & 70 \\
VIH (\%) & 0 \\
Etapa IV (\%) & 80 \\
Compromiso MO (\%) & 80 \\
Compromiso SNC (\%) & 0 \\
Ki67 (mediana; rango) & $20 ; 10-60$ \\
MIPI (\%) & 20 \\
- Bajo & 35 \\
- Intermedio & 45 \\
- Alto & \\
Tratamiento primera línea (\%) & 25 \\
- R-HyperCVAD & 55 \\
- R-CHOP & 5 \\
- R-COP & 5 \\
- Cirugía & 10 \\
- Observación & 10 \\
TPH en primera línea (\%) & \\
\hline
\end{tabular}

Abreviaciones: DS: desviación estándar; Ki67: antígeno relacionado con índice de proliferación; LM: Linfoma del manto; MIPI: Mantle cell lymphoma International Prognostic Index; MO: médula ósea; R-CHOP: rituximab + ciclofosfamida + doxorrubicina + vincristina + prednisona; R-COP: rituximab + ciclofosfamida + vincristina + prednisona; R-HyperCVAD: rituximab + ciclofosfamida + vincristina + doxorrubicina + dexametasona alternado con metotrexato + citarabina; SNC: sistema nervioso central; TPH: trasplante de precursores hematopoyéticos; VIH: virus de la inmunodeficiencia humana.
En primera línea, 25\% $(n=5)$ recibió quimioterapia intensiva basada en AraC con esquema R-HyperCVAD (rituximab, ciclofosfamida, vincristina, doxorrubicina, dexametasona alternado con metotrexato y AraC) y 55\% $(\mathrm{n}=11)$ recibió R-CHOP. De los 4 pacientes restantes, 1 recibió $\mathrm{R}$-COP, 1 fue tratada con resección de una lesión cutánea con estudio sistémico negativo, y 2 fueron observados sin iniciarse tratamiento por clínica indolente.

\section{Respuesta a tratamiento y sobrevida}

Las características iniciales de los pacientes, esquemas de tratamiento de primera línea, respuesta a tratamiento y sobrevida se detallan en la Tabla 2. En los pacientes tratados con quimioterapia, la tasa de respuesta global $(\mathrm{RG}=\mathrm{RC}+\mathrm{RP})$ fue $76,5 \%(n=13)$. La diferencia en las tasas de $\mathrm{RC}$ entre esquemas no fue significativa, siendo $60 \%$ para R-HyperCVAD y $45,5 \%$ para R-CHOP $(p=0,59)$. Los pacientes en etapa IV tuvieron significativamente menor probabilidad de lograr $\mathrm{RC}$ con quimioterapia en comparación con etapas más tempranas ( $100 \%$ en etapas I a III vs $31,3 \%$ en etapa IV; $p=0,026)$ y de forma similar, aunque sin lograr significancia, cuando el índice pronóstico MIPI fue alto también hubo menos probabilidad de lograr RC (66,7\% para MIPI bajo o intermedio vs $25 \%$ para MIPI alto; $\mathrm{p}=0,085)$. Durante la evolución, $60 \%$ de los pacientes presentó al menos una recaída o fue primariamente refractario. En ellos, se indicaron en total 27 tratamientos de rescate, logrando respuesta en la mayoría de los casos ( $R G 77,7 \%, n=21)$ (Tabla 3 ). Por otro lado, durante su evolución, 6 pacientes fueron sometidos a TPH, cuyos condicionamientos fueron en 2 casos con BEAM (carmustina, etopósido, citarabina y melfalán), en 3 con BEC (busulfán, etopósido y ciclofosfamida) y en 1 con Cy-FluTBI-Cy (ciclofosfamida, fludarabina, irradiación corporal total).

A 4 años, la SLP de los pacientes con LM fue de $29,5 \%$, con una mediana de 33,3 meses. La SG a 4 años fue de $77,4 \%$, con una mediana de 130 meses (Figura 1). El índice MIPI discriminó significativamente la probabilidad de progresar y fallecer. Así, la SLP a 4 años fue de 100\%, 51,4\% y $11 \%$ para MIPI bajo, intermedio y alto, respectivamente $(\mathrm{p}=0,003)$, mientras que la SG a 4 años fue de $100 \%$ para MIPI bajo e intermedio y $55,5 \%$ para MIPI alto $(p=0,02)$, con mediana de 73 
Tabla 2. Resultados de tratamiento de primera línea de pacientes con linfoma del manto

\begin{tabular}{|c|c|c|c|c|c|c|c|c|c|c|}
\hline $\mathbf{N}$ & Edad & Sexo & Etapa & MIPI & Protocolo & Respuesta & Mantención & $\begin{array}{c}\text { TPH } \\
1^{\circ}\end{array}$ & $\begin{array}{c}\text { SLP } \\
\text { meses }\end{array}$ & $\begin{array}{c}\text { SG } \\
\text { meses }\end{array}$ \\
\hline 1 & 54 & M & 2 & B & R-HyperCVAD & $\mathrm{RC}$ & No & Sí & 63,7 & $87,4^{* *}$ \\
\hline 2 & 55 & M & 4 & I & R-HyperCVAD & $\mathrm{RC}$ & Sí & No & $63,1^{*}$ & $63,1^{* *}$ \\
\hline 3 & 40 & $\mathrm{~F}$ & 4 & $A$ & R-HyperCVAD & $\mathrm{RC}$ & No & Sí & 4,8 & 4,8 \\
\hline 4 & 45 & M & 4 & $A$ & R-HyperCVAD & $E R$ & No & No & 2,6 & $42,8^{* *}$ \\
\hline 5 & 67 & M & 4 & $A$ & R-HyperCVAD & $E R$ & No & No & 2,0 & $75,7^{* *}$ \\
\hline 6 & 64 & $\mathrm{~F}$ & 4 & 1 & $\mathrm{R}-\mathrm{CHOP}$ & $\mathrm{RP}$ & Sí & No & $66,9^{*}$ & $66,9^{* *}$ \\
\hline 7 & 64 & M & 1 & 1 & $\mathrm{R}-\mathrm{CHOP}$ & $\mathrm{RC}$ & Sí & No & $61,4^{*}$ & $61,4^{* *}$ \\
\hline 8 & 60 & M & 4 & A & $\mathrm{R}-\mathrm{CHOP}$ & $\mathrm{RP}$ & Sí & No & 48,1 & 130,3 \\
\hline 9 & 57 & M & 4 & 1 & $\mathrm{R}-\mathrm{CHOP}$ & $\mathrm{RC}$ & No & No & 40,5 & $133,0^{* *}$ \\
\hline 10 & 67 & $\mathrm{~F}$ & 4 & 1 & $\mathrm{R}-\mathrm{CHOP}$ & $\mathrm{RC}$ & Sí & No & 33,3 & $133,9 * *$ \\
\hline 11 & 60 & M & 4 & B & $\mathrm{R}-\mathrm{CHOP}$ & $\mathrm{RP}$ & Sí & No & $28,6^{*}$ & $28,6^{* *}$ \\
\hline 12 & 44 & M & 3 & B & $\mathrm{R}-\mathrm{CHOP}$ & $\mathrm{RC}$ & Sí & No & $26,5^{*}$ & $26,5^{* *}$ \\
\hline 13 & 66 & M & 4 & $A$ & $\mathrm{R}-\mathrm{CHOP}$ & $\mathrm{RP}$ & Sí & No & 21,4 & $46,5^{* *}$ \\
\hline 14 & 71 & M & 4 & A & $\mathrm{R}-\mathrm{CHOP}$ & $\mathrm{RC}$ & Sí & No & 18,0 & 23,1 \\
\hline 15 & 62 & $\mathrm{~F}$ & 4 & 1 & $\mathrm{R}-\mathrm{CHOP}$ & ER & No & No & 4,0 & $30,4^{* *}$ \\
\hline 16 & 64 & $\mathrm{M}$ & 4 & A & $\mathrm{R}-\mathrm{CHOP}$ & $E R$ & No & No & 1,9 & 73,0 \\
\hline 17 & 79 & $\mathrm{~F}$ & 4 & A & R-COP & $\mathrm{RP}$ & Sí & No & 10,4 & 11,2 \\
\hline 18 & 62 & $\mathrm{~F}$ & 1 & B & Cirugía & $\mathrm{RC}$ & No & No & $7,5^{*}$ & $7,5^{* *}$ \\
\hline 19 & 84 & M & 4 & A & Observación & $(-)$ & No & No & 32,1 & 39,9 \\
\hline 20 & 72 & M & 4 & 1 & Observación & $(-)$ & No & No & $25,6^{*}$ & $25,6^{* *}$ \\
\hline
\end{tabular}

Notas: *Continúa en seguimiento sin progresar ni fallecer. ${ }^{*}$ Continúa en seguimiento sin fallecer. Abreviaciones: ER, enfermedad refractaria; F: femenino; M: masculino; MIPI: Mantle cell lymphoma International Prognostic Index (B: bajo; I: intermedio; A: alto); R-CHOP: rituximab + ciclofosfamida + doxorrubicina + vincristina + prednisona; R-COP: rituximab + ciclofosfamida + vincristina + prednisona; R-HyperCVAD: rituximab + ciclofosfamida + vincristina + doxorrubicina + dexametasona alternado con metotrexato + citarabina; RC: respuesta completa; RP: respuesta parcial; SG: sobrevida global; SLP: sobrevida libre de progresión; TPH 1: trasplante de precursores hematopoyéticos en primera línea.

meses en este último caso (Figura 2). En el análisis univariado, no hubo diferencias en sobrevida de acuerdo al esquema de quimioterapia utilizado en primera línea, tanto en SLP (R-HyperCVAD 40\% vs R-CHOP 38,2\% a 4 años; $\mathrm{p}=0,65)$, como en SG (R-HyperCVAD $80 \%$ vs R-CHOP $90,9 \%$ a 4 años, $\mathrm{p}=0,91)$.

\section{Análisis de subgrupos}

En los pacientes tratados con quimioterapia que lograron respuesta parcial o superior (34 de los 44 tratamientos), posteriormente, se siguieron distintas conductas; mantención basada en rituxi- mab en 16 casos, TPH en 6 , o ningún otro tratamiento en los 12 restantes. Pacientes expuestos a mantención y/o TPH tuvieron significativamente mayor SLP $(58,3 \%$ vs $18,3 \%$ a 3 años, con tiempo libre de recaída de 48,1 vs 21,2 meses ( $\mathrm{p}=0,003$ ) (Figura 3). Este aumento en SLP solo se observó cuando se analizaron mantención y TPH agrupadamente, no existiendo diferencias significativas al analizarlos por separado.

De todos los pacientes tratados con quimioterapia, se observó un aumento no significativo en SG a 4 años para quienes recibieron AraC en alguna línea o TPH ( $\mathrm{n}=9$, SG $88,9 \%$ vs $62,2 \%$, 
Resultados en tratamiento de linfoma del manto UC - J. Campbell et al

Tabla 3. Tratamientos de rescate en pacientes con linfoma del manto

\begin{tabular}{|c|c|c|c|c|}
\hline Esquema & $\mathbf{n}$ & RG (\%) & SLP (meses) & SLP a 2 años (\%) \\
\hline FCR & 3 & 66,7 & 44,3 & 66,7 \\
\hline Obinutuzumab & 2 & 100 & 24,5 & 50 \\
\hline R-BAC & 1 & 100 & 29,7 & 100 \\
\hline R-Bendamustina & 2 & 100 & 21,2 & 50 \\
\hline $\mathrm{R}-\mathrm{COP}$ & 4 & 75 & 10,8 & 0 \\
\hline R-DHAP & 1 & 100 & NA & SI \\
\hline R-HyperCVAD & 1 & 0 & 3 & 0 \\
\hline R-ICE & 1 & 100 & 27,7 & 100 \\
\hline R-Lenalidomida & 2 & 100 & NA & $\mathrm{SI}$ \\
\hline R-MINE & 2 & 50 & 16,1 & 50 \\
\hline R-VCD & 4 & 75 & 36,9 & 50 \\
\hline $\mathrm{TPH} 2^{\circ}$ o mayor & 4 & 100 & NA & 100 \\
\hline VCD & 1 & 100 & 64,4 & 100 \\
\hline VRD & 3 & 66,7 & 9,5 & 0 \\
\hline
\end{tabular}

Abreviaciones: FCR: fludarabina + ciclofosfamida + rituximab; NA: no alcanzada; R-BAC: rituximab + bendamustina + citarabina; R-bendamustina: rituximab + bendamustina; R-COP: rituximab + ciclofosfamida + vincristina + prednisona; R-DHAP: rituximab + dexametasona + citarabina + cisplatino; RG: respuesta global; R-HyperCVAD: rituximab + ciclofosfamida + vincristina + doxorrubicina + dexametasona alternado con metotrexato + citarabina; R-ICE: rituximab + ifosfamida + carboplatino + etopósido; R-lenalidomida: rituximab + lenalidomida; R-MINE: rituximab + mesna + ifosfamida + novantrona + etopósido; R-VCD: rituximab + bortezomib + ciclofosfamida + dexametasona; SI: seguimiento insuficiente; SLP: sobrevida libre de progresión; TPH $2^{\circ}$ o mayor: trasplante de precursores hematopoyéticos en $2^{\circ}$ línea o posterior; VCD: bortezomib, ciclofosfamida, dexametasona; VRD: bortezomib, rituximab, dexametasona.

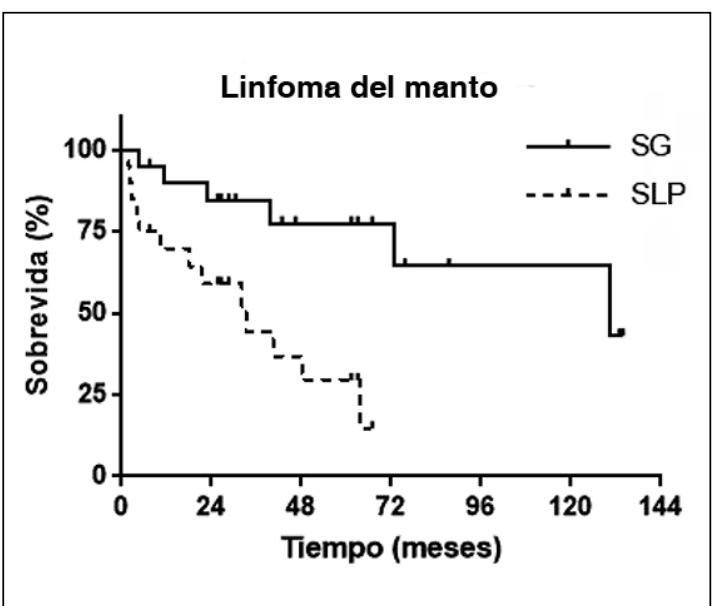

Figura 1. Sobrevida LM. LM: linfoma del manto; SG: sobrevida global; SLP: sobrevida libre de progresión. $\mathrm{p}=0,38)$. De los 12 pacientes que fueron refractarios a primera línea o recayeron, los expuestos a AraC presentaron un aumento significativo en SLP $(27,5$ vs 9,3 meses, $p=0,044)$ y no significativo en SG. De igual forma, aquellos que recibieron TPH tuvieron mejoría no significativa de SLP (33,5 vs 15,6 meses, $p=0,078)$. El efecto de ambos tratamientos fue aditivo, dado que los pacientes sometidos a AraC, TPH o ambos tuvieron aumento significativo tanto en SLP $(27,5$ vs $9,3$ meses, $\mathrm{p}=0,02)$ como en SG $(100 \%$ vs $40 \%$ a 4 años, mediana $>133$ vs 39,9 meses, $\mathrm{p}=0,046$ ) (Figura 4). En este subgrupo de pacientes el beneficio fue especialmente marcado en aquellos con MIPI alto, quienes lograron SLP de 25,9 vs 3,8 meses $(\mathrm{p}=0,023)$ y SG de $100 \%$ vs $25 \%$ a 4 años $(\mathrm{p}=0,041)$. 


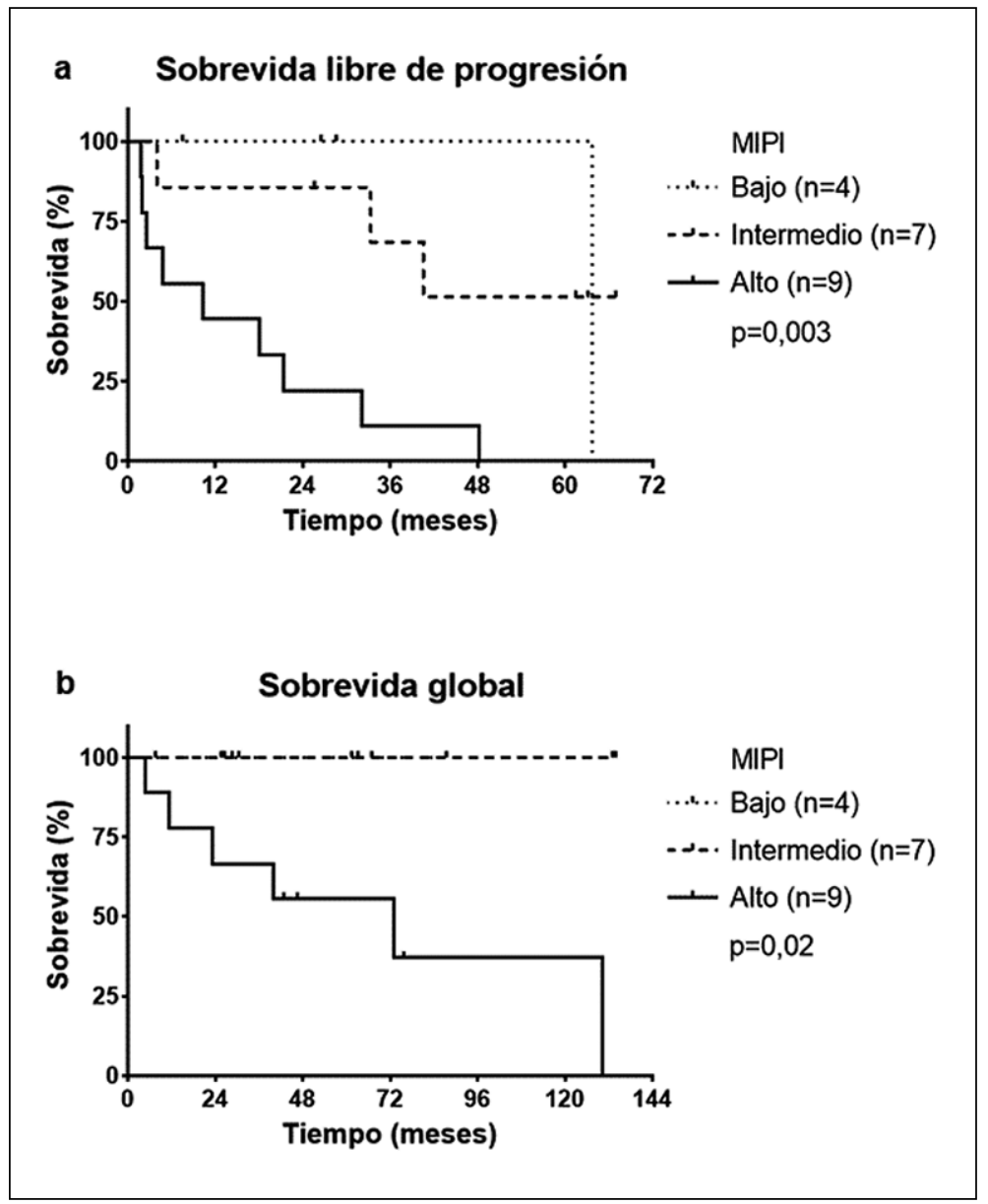

Figura 2. Pronóstico LM según Índice de Riesgo -MIPI-. LM: linfoma del manto; MIPI: Mantle cell lymphoma International Prognostic Index.

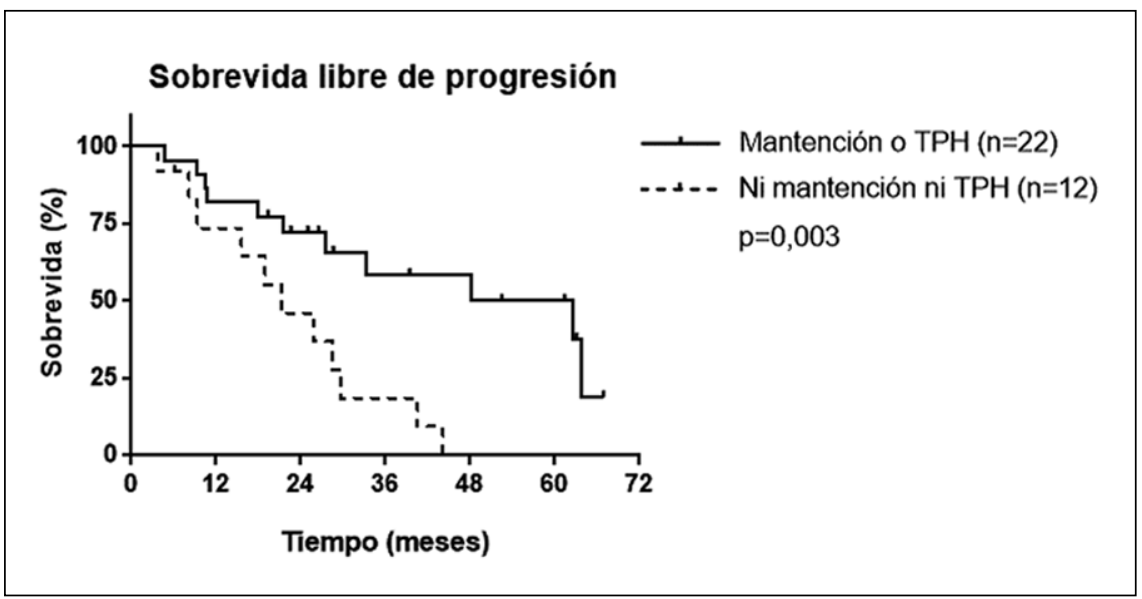

Figura 3. Impacto de mantención y TPH en sobrevida de pacientes con LM. LM:linfoma del manto; $\mathrm{TPH}$ : trasplante de precursores hematopoyéticos. 


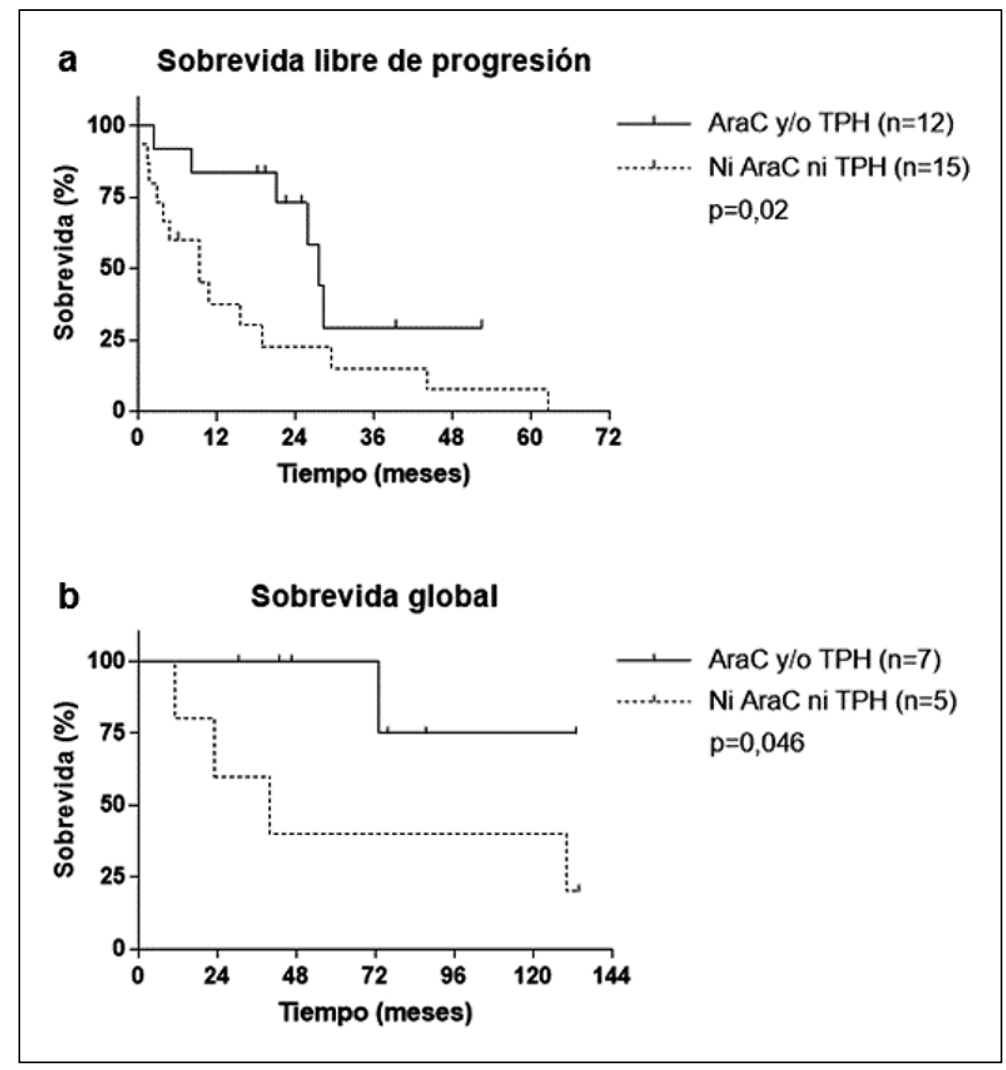

Figura 4. Exposición a AraC, TPH O ambos. Análisis de pacientes con enfermedad recaída o refractaria, exclusivamente. AraC: citarabina; TPH: trasplante de precursores hematopoyéticos.

\section{Discusión}

El presente estudio es la primera publicación sobre resultados del tratamiento de pacientes adultos chilenos con linfoma del manto. Pese a sus limitaciones, nuestros resultados están en sintonía con los principales ensayos clínicos internacionales, que apoyan el uso de terapia intensificada y mantención con rituximab.

El primer estudio aleatorizado que evaluó la utilidad del TPH en LM demostró que consolidar la respuesta a tratamiento con TPH luego de CHOP (sin rituximab) aumenta la SLP casi 2 años ( 39 vs 17 meses, $\mathrm{p}=0,0108$ ), sin cambios en SG a 3 años $(83 \% \text { vs } 77 \% ; p=0,18)^{12}$. El uso de AraC también se asocia a un aumento en la SLP, así, al tratar pacientes con R-HyperCVAD/MA, 97\% responde y $87 \%$ logra $\mathrm{RC}$, traduciéndose en una SLP a 3 años de $73 \%{ }^{13}$. De forma similar, el grupo nórdico usó un esquema de inducción basado en $\mathrm{CHOP}$ alternado con AraC, seguido de rituximab y TPH con $\mathrm{AraC}^{14}$. En estos pacientes, la SLP fue de 7,4 años y la SG no fue alcanzada con más de 10 años de seguimiento. El estudio fase 3 más reciente incluyó 455 pacientes y comparó R-CHOP seguido de TPH versus R-CHOP alternado con R-DHAP (incluye AraC) seguido de TPH con $\mathrm{AraC}^{17}$. El grupo que recibió AraC tuvo un aumento significativo en SLP (9,1 vs 4,3 años, $\mathrm{p}<0,0001)$ y un aumento en SG a 5 años que no logró significancia $(76 \%$ vs $69 \%$; HR 0,78, IC 95\% 0,57-1,07, $\mathrm{p}=0,12$ ). Pese a que los dos estudios fase 3 aleatorizados no lograsen demostrar significancia en el aumento de SG, es notable que a medida que se intensifica la terapia, especialmente al combinarse TPH y AraC en altas dosis, los pacientes logran medianas de SG que se acercan a los 10 años. Finalmente, replicando el beneficio en LNH indolentes, el uso de rituximab como terapia de mantención también ha demostrado beneficio en el LM. En pacientes tratados con R-CHOP, recibir mantención con rituximab se asocia a un aumento significativo en 
la SG a 4 años $(87 \% \text { vs } 63 \%, p=0,005)^{26}$. Además, el beneficio de la mantención es aditivo al de la terapia intensiva con AraC y TPH ${ }^{18,27}$.

Pese a las características de nuestro estudio, los resultados muestran que incluso con un bajo número de pacientes analizables y baja tasa de $\mathrm{TPH}$ en primera línea, el impacto de AraC, TPH y mantención con rituximab es significativo. Tratados con R-CHOP, los pacientes logran RC en 45,5\% de los casos; si no se indica TPH ni mantención, a 3 años, $81,7 \%$ habrá recaído. Por otro lado, si luego de cualquier quimioterapia reciben $\mathrm{TPH}$, mantención con rituximab o ambos, más de la mitad $(58,3 \%)$ seguirá con respuesta a 3 años, recayendo 26,9 meses después que aquellos que no reciben ninguna terapia adicional. Igualmente, todos los pacientes en recaída que reciben AraC, TPH o ambos están vivos a 4 años, mientras que en ausencia de estas terapias, solo $40 \%$ lo está. En general, cualquier otra terapia en recaída logra una respuesta menor a un año, mientras que AraC y TPH logran respuestas superiores a los 2 años. Más aun, aunque no fue evaluado de manera dirigida por el bajo $\mathrm{n}$ de pacientes, el uso de regímenes de condicionamiento de trasplante basados en citarabina (BEAM), pudiese incluso aumentar la respuesta en comparación con otros esquemas que no contienen citarabina. En suma, considerando a todos los pacientes con LM estudiados, la mediana de SG supera los 10 años (130 meses), muy por sobre las expectativas históricas con tratamiento tradicional.

En Chile, aún el plan de Acceso Universal de Garantías Explícitas (AUGE) considera y garantiza únicamente R-CHOP para el tratamiento de $\mathrm{LM}^{28}$. Recientemente, la Sociedad Chilena de Hematología (SOCHIHEM) publicó una guía para el diagnóstico y tratamiento de $\mathrm{LNH}^{29}$, la cual, por primera vez, manifiesta la importancia que tienen esquemas basados en AraC y el TPH, en especial para pacientes menores de 65 años (>50\% de los pacientes) y aquellos con alto riesgo según MIPI. Nuestros resultados apoyan localmente las recomendaciones de la SOCHIHEM.

En conclusión, los pacientes con LM tienen aumento significativo de sobrevida cuando en su tratamiento se incluyen esquemas de alta intensidad, combinando protocolos con AraC y TPH, $\mathrm{y}$ adicionalmente mantención con rituximab. El efecto de estas estrategias es sinérgico y de particular beneficio para los pacientes de mayor riesgo según el índice MIPI. Sugerimos considerar el presente estudio para el desarrollo de futuras guías de manejo de LNH.

Agradecimientos: A Ximena Solar e Isabel Rodríguez por su contribución en la recolección de los datos; y al equipo multidisciplinario de Hematología de la Red de Salud UC-Christus por la constante preocupación por el mejor cuidado de nuestros pacientes.

\section{Referencias}

1. Mozos A, Royo C, Hartmann E, De Jong D, Baro C, Valera A, et al. SOX11 expression is highly specific for mantle cell lymphoma and identifies the cyclin D1-negative subtype. Haematologica 2009; 94 (11): 1555-62.

2. Campo E, Rule S. Mantle cell lymphoma: evolving management strategies. Blood 2015; 125 (1): 48-55.

3. Cabrera ME, Martínez V, Nathwani BN, Muller-Hermelink HK, Diebold J, Maclennan KA, et al. Non-Hodgkin lymphoma in Chile: a review of 207 consecutive adult cases by a panel of five expert hematopathologists. Leuk Lymphoma 2012; 53 (7): 1311-7.

4. Leux C, Maynadie M, Troussard X, Cabrera Q, Herry A, Le Guyader-Peyrou S, et al. Mantle cell lymphoma epidemiology: a population-based study in France. Ann Hematol 2014; 93 (8): 1327-33.

5. Cheah CY, Seymour JF, Wang ML. Mantle Cell Lymphoma. J Clin Oncol 2016; 34 (11): 1256-69.

6. Medina E, Kaempffer A. [Trends and main features of Chilean mortality]. Rev Med Chile 2007; 135 (2): 240-50.

7. Campo E, Swerdlow SH, Harris NL, Pileri S, Stein H, Jaffe ES. The 2008 WHO classification of lymphoid neoplasms and beyond: evolving concepts and practical applications. Blood 2011; 117 (19): 5019-32.

8. Jaffe ES, Pittaluga S. Aggressive B-cell lymphomas: a review of new and old entities in the WHO classification. Hematology Am Soc Hematol Educ Program 2011; 2011: 506-14.

9. Cortelazzo S, Ponzoni M, Ferreri AJ, Dreyling M. Mantle cell lymphoma. Crit Rev Oncol Hematol 2012; 82 (1): 78-101.

10. Fisher RI, Gaynor ER, Dahlberg S, Oken MM, Grogan TM, Mize EM, et al. Comparison of a standard regimen ( $\mathrm{CHOP}$ ) with three intensive chemotherapy regimens for advanced non-Hodgkin's lymphoma. N Engl J Med 1993; 328 (14): 1002-6.

11. Lenz G, Dreyling M, Hoster E, Wormann B, Duhrsen U, Metzner B, et al. Immunochemotherapy with rituximab 
and cyclophosphamide, doxorubicin, vincristine, and prednisone significantly improves response and time to treatment failure, but not long-term outcome in patients with previously untreated mantle cell lymphoma: results of a prospective randomized trial of the German Low Grade Lymphoma Study Group (GLSG). J Clin Oncol 2005; 23 (9): 1984-92.

12. Dreyling M, Lenz G, Hoster E, Van Hoof A, Gisselbrecht C, Schmits R, et al. Early consolidation by myeloablative radiochemotherapy followed by autologous stem cell transplantation in first remission significantly prolongs progression-free survival in mantle-cell lymphoma: results of a prospective randomized trial of the European MCL Network. Blood 2005; 105 (7): 2677-84.

13. Romaguera JE, Fayad L, Rodríguez MA, Broglio KR, Hagemeister FB, Pro B, et al. High rate of durable remissions after treatment of newly diagnosed aggressive mantle-cell lymphoma with rituximab plus hyper-CVAD alternating with rituximab plus high-dose methotrexate and cytarabine. J Clin Oncol 2005; 23 (28): 7013-23.

14. Geisler CH, Kolstad A, Laurell A, Jerkeman M, Raty R, Andersen NS, et al. Nordic MCL2 trial update: six-year follow-up after intensive immunochemotherapy for untreated mantle cell lymphoma followed by BEAM or BEAC + autologous stem-cell support: still very long survival but late relapses do occur. Br J Haematol 2012; 158 (3): 355-62.

15. Merli F, Luminari S, Ilariucci F, Petrini M, Visco C, Ambrosetti A, et al. Rituximab plus HyperCVAD alternating with high dose cytarabine and methotrexate for the initial treatment of patients with mantle cell lymphoma, a multicentre trial from Gruppo Italiano Studio Linfomi. Br J Haematol 2012; 156 (3): 346-53.

16. Delarue R, Haioun C, Ribrag V, Brice P, Delmer A, Tilly $\mathrm{H}$, et al. CHOP and DHAP plus rituximab followed by autologous stem cell transplantation in mantle cell lymphoma: a phase 2 study from the Groupe d'Etude des Lymphomes de l'Adulte. Blood 2013; 121 (1): 4853.

17. Hermine O, Hoster E, Walewski J, Bosly A, Stilgenbauer S, Thieblemont C, et al. Addition of high-dose cytarabine to immunochemotherapy before autologous stem-cell transplantation in patients aged 65 years or younger with mantle cell lymphoma (MCL Younger): a randomised, open-label, phase 3 trial of the European Mantle Cell Lymphoma Network. The Lancet 2016; 388 (10044): 565-75.

18. Le Gouill S, Thieblemont C, Oberic L, Moreau A,
Bouabdallah K, Dartigeas C, et al. Rituximab after Autologous Stem-Cell Transplantation in Mantle-Cell Lymphoma. N Engl J Med 2017; 377 (13): 1250-60.

19. Goy A, Bernstein SH, Kahl BS, Djulbegovic B, Robertson MJ, de Vos S, et al. Bortezomib in patients with relapsed or refractory mantle cell lymphoma: updated time-toevent analyses of the multicenter phase 2 PINNACLE study. Ann Oncol 2009; 20 (3): 520-5.

20. Trněný M, Lamy T, Walewski J, Belada D, Mayer J, Radford J, et al. Lenalidomide versus investigator's choice in relapsed or refractory mantle cell lymphoma (MCL-002; SPRINT): a phase 2 , randomised, multicentre trial. The Lancet Oncology 2016; 17 (3): 319-31.

21. Dreyling M, Jurczak W, Jerkeman M, Silva RS, Rusconi C, Trneny M, et al. Ibrutinib versus temsirolimus in patients with relapsed or refractory mantle-cell lymphoma: an international, randomised, open-label, phase 3 study. The Lancet 2016; 387 (10020): 770-8.

22. Dreyling M, Campo E, Hermine O, Jerkeman M, Le Gouill S, Rule S, et al. Newly diagnosed and relapsed mantle cell lymphoma: ESMO Clinical Practice Guidelines for diagnosis, treatment and follow-up. Ann Oncol 2017; 28 (suppl_4): iv62-iv71.

23. Swerdlow SH, Campo E, Harris NL, Jaffe ES, Pileri SA, Stein H, et al. WHO Classification of Tumours of Haematopoietic and Lymphoid Tissues, Fourth Edition. IARC, editor: WHO; 2008.

24. Cheson BD, Fisher RI, Barrington SF, Cavalli F, Schwartz LH, Zucca E, et al. Recommendations for initial evaluation, staging, and response assessment of Hodgkin and non-Hodgkin lymphoma: the Lugano classification. J Clin Oncol 2014; 32 (27): 3059-68.

25. Cunningham J, Iyengar S, Sharma B. Evolution of lymphoma staging and response evaluation: current limitations and future directions. Nat Rev Clin Oncol 2017; 14 (10): 631-45.

26. Kluin-Nelemans HC, Hoster E, Hermine O, Walewski J, Trneny M, Geisler CH, et al. Treatment of older patients with mantle-cell lymphoma. N Engl J Med 2012; 367 (6): 520-31.

27. Ahmadi T, McQuade J, Porter D, Frey N, Loren AW, Goldstein SC, et al. Potential prolongation of PFS in mantle cell lymphoma after R-HyperCVAD: auto-SCT consolidation or rituximab maintenance. Bone Marrow Transplant 2012; 47 (8): 1082-6.

28. MINSAL. Guía Clínica AUGE LINFOMA en personas de 15 años y más. www.bibliotecaminsal.cl2013.

29. SOCHIHEM. Diagnóstico y Tratamiento de Linfomas No Hodgkin. www.sochihem.cl; 2017. 